\title{
Factors influencing practice variation in the management of nephrotic syndrome: a qualitative study of pediatric nephrology care providers
}

\author{
Susan M. Samuel MD MSc, Rachel Flynn MScN RN, Michael Zappitelli MD MSc, Allison Dart MD MSc, \\ Rulan Parekh MD MSc, Maury Pinsk MD MSc, Cherry Mammen MD MSc, Andrew Wade MD PhD, \\ Shannon D. Scott PhD RN; for the Canadian Childhood Nephrotic Syndrome Project Team*
}

\section{Abstract}

Background: Treatment protocols for childhood nephrotic syndrome are highly variable between providers and care centres. We conducted a qualitative study to understand the complex multilevel processes that lead to practice variation and influence provider management of nephrotic syndrome.

Methods: Focus groups with multidisciplinary pediatric nephrology care providers $(n=67)$ from 10 Canadian pediatric nephrology centres that had more than 1 pediatric nephrologist were conducted between September 2013 and April 2015. Focus group discussions were guided by the Ottawa Model for Research Use. We used a semistructured interview guide to elicit participants' perspectives regarding 1) the work setting and context of the clinical environment, 2) reasons for variation at the provider level and 3) clinical practice guidelines for nephrotic syndrome. Focus group discussions were transcribed and analyzed concurrently with the use of qualitative content analysis.

Results: Emerging themes were grouped into 2 categories: centre-level factors and provider-level factors. At the centre level, the type of care model used, clinic structures and resources, and lack of communication and collaboration within and between Canadian centres influenced care variation. At the provider level, use of experiential knowledge versus empirical knowledge and interpretation of patient characteristics influenced provider management of nephrotic syndrome.

Interpretation: Centre- and provider-level factors play an important role in shaping practice differences in the management of childhood nephrotic syndrome. Further research is needed to determine whether variation in care is associated with disparities in outcomes.

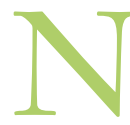

ephrotic syndrome affects 16 per 100000 children worldwide. ${ }^{1}$ Treatment of relapsing childhood nephrotic syndrome includes glucocorticoids as first-line therapy, but multiple drugs and courses of therapy over long periods may also be required to prevent relapses. ${ }^{2}$ International clinical practice guidelines are available to guide treatment of childhood nephrotic syndrome; ${ }^{3}$ however, many recommendations are based on few randomized controlled trials in this rare disease, and available drugs have substantial toxic effects. ${ }^{4,5}$ Treatment protocols and processes of care for childhood nephrotic syndrome are also highly variable between providers and centres. ${ }^{6,7}$ Lack of evidence to guide therapeutic decisions may cause significant anxiety and dissatisfaction with care among patients and families. ${ }^{8,9}$
Understanding why there is practice variation is a key step to overcoming barriers to the implementation of standardized approaches based on available evidence across many centres. The aim of this qualitative study was to provide an understanding of the complex multilevel processes that lead to practice variation and influence provider

\section{Competing interests: None declared.}

*The list of members appears at the end of the article.

This article has been peer reviewed.

Correspondence to: Susan Samuel, susan.samuel@ahs.ca

CMAJ Open 2017. DOI:10.9778/cmajo.20160078 
decision-making in the treatment of childhood nephrotic syndrome.

\section{Methods}

\section{Design}

\section{Focus groups}

We conducted focus groups grounded in the Ottawa Model of Research Use ${ }^{10}$ between September 2013 and April 2015 with pediatric nephrologists and multidisciplinary health care providers who care for children with nephrotic syndrome (e.g., nurses, pharmacists, dieticians) to explore the reasons for treatment variation. The Ottawa Model of Research Use offers a comprehensive, interdisciplinary framework of elements that affect the process of transfer of health care knowledge. It is derived from theories of change, published literature and reflection. ${ }^{10,11}$

Focus group approaches capture multiple perspectives (e.g., beliefs, values, actions and behaviours), including the complexity of group dynamics and hierarchy, in a timeefficient manner. ${ }^{12}$ The discussion that occurs in focus groups allows participants to ask questions of each other (e.g., "How do you decide to taper steroids in this situation?" and "Which second-line agent would you choose in which patient?") as well as reevaluate and reconsider their own perspectives. ${ }^{13}$ This level of reflection and discussion is particularly useful in exploring the complexity of factors contributing to practice variation within centres. In keeping with qualitative approaches, ${ }^{14}$ we did not specify any a priori hypothesis.

\section{Setting and participants}

We conducted 1 focus group per pediatric nephrology centre across 10 pediatric nephrology centres in Canada (4 centres in western Canada [British Columbia and Alberta], 4 centres in Ontario and 2 centres in Quebec). There are 13 pediatric nephrology centres in Canada, and we purposefully selected centres that had more than 1 pediatric nephrologist $(n=10)$. Pediatric nephrologists, nurse specialists and multidisciplinary health care providers who care for children with nephrotic syndrome in Canadian tertiary pediatric nephrology centres (with more than 1 pediatric nephrologist providing service) and associated satellite clinics were eligible for recruitment. We identified a lead physician most involved in nephrotic syndrome care at each centre a priori, and these physicians identified eligible key informants (physicians, nurses, dieticians, social workers, other multidisciplinary professionals) at their centre and informed these people about the study. Eligible participants were approached face to face by the lead physician before the focus group, and the focus group was scheduled at a time when all those caring for patients with nephrotic syndrome were available. The lead physician was involved in recruiting key informants as appropriate for his or her clinic. Eligible participants were purposefully identified as information-rich participants regarding the phenomenon of interest. To protect the identity of the people approached, we did not record the number of people who were approached or who declined.

\section{Interview guide}

The interview guide for the focus groups was pilot tested at the lead researcher's (S.M.S., a pediatric nephrologist) site. Iterations to the interview guide were based on responses and feedback at this pilot site.

The semistructured interview guide was divided into 3 parts: 1) understanding the work setting and context of the clinical environment to explore how patient-, unit- or centrelevel factors may influence treatment decisions, 2) identifying reasons for variation at the provider level and 3) understanding the participants' perceptions regarding clinical practice guidelines for nephrotic syndrome (see Appendix 1, available at www.cmajopen.ca/content/5/2/E424/suppl/DC1). All of the focus groups were conducted in English and were moderated by a qualitative researcher (R.F.) who is an $\mathrm{RN} \mathrm{PhD} \mathrm{can-}$ didate trained in qualitative research by her $\mathrm{PhD}$ supervisor (S.D.S.), a qualitative researcher. S.D.S. was not present at the interviews but assisted with interview guide development. S.M.S. trained R.F. in aspects of nephrotic syndrome management. R.F. was not known to the participants in advance of the focus group. S.M.S. was known to many of the pediatric nephrology physicians before the study. At the start of each focus group, S.M.S. presented her reasons for her interest in the research topic (to improve care and outcomes of children with nephrotic syndrome). During the focus groups, S.M.S. recorded field notes (descriptive written accounts of events capturing nuances in conversation and group dynamics); she did not contribute to the focus group discussion.

To identify areas of practice variation, participants were asked to talk about their general approach to management of initial presentation of nephrotic syndrome, followed by openended questions regarding relapses, second-line therapy and use of kidney biopsy. We selected these topics based on a prior survey in which we evaluated practice patterns of Canadian pediatric nephrologists. ${ }^{7}$ The key informants were asked to provide details that underpinned their treatment decisions. We purposefully collected data from all high-volume Canadian pediatric hospitals for nephrotic syndrome to get a wide representation from our data.

\section{Transcription}

All of the focus groups were audiorecorded. A stenographer performed real-time transcription verbatim, an approach that prevents loss of important contextual information and provides a more complete account of the proceedings on which to base subsequent analysis. ${ }^{15}$

\section{Analysis}

Data collection and analysis occurred concurrently with the use of qualitative thematic content analysis. ${ }^{16}$ The essence of this analytic approach is to identify patterns in the data that are associated with the research question. First, all data were coded to facilitate analysis. The codes were then operationally defined so that they could be applied consistently throughout 


\section{OPEN}

the data. We developed a hierarchical arrangement of coding using NVivo 10 software (QSR International). Second, coders R.F. and S.M.S. discussed the initial codes and placed them into broad categories that corresponded to the major unit of analysis. As categories emerged, their theoretical properties were defined. Comparisons between multiple categories were carried out to locate similarities and differences. Finally, these categories were synthesized into themes. R.F. and S.D.S. met regularly to discuss the emerging analysis. Data saturation was

\begin{tabular}{|c|c|}
\hline Characteristic & $\begin{array}{c}\text { No. }(\%) \text { of } \\
\text { participants } \\
n=67\end{array}$ \\
\hline No. of participants per site, median (range) & $6(4-11)$ \\
\hline \multicolumn{2}{|l|}{ Discipline } \\
\hline Physician & $42(63)$ \\
\hline Nurse & $15(22)$ \\
\hline Allied health care worker & $6(9)$ \\
\hline Dietician/pharmacist/social worker & $4(6)$ \\
\hline \multicolumn{2}{|l|}{ Age, yr† } \\
\hline$<40$ & $8(12)$ \\
\hline $40-50$ & $25(37)$ \\
\hline $51-60$ & $12(18)$ \\
\hline$>60$ & $5(7)$ \\
\hline Unknown & $17(25)$ \\
\hline \multicolumn{2}{|l|}{ Length of experience in discipline, yr† } \\
\hline$<10$ & $11(16)$ \\
\hline $10 \leq 20$ & $24(36)$ \\
\hline$>20$ & $16(24)$ \\
\hline Unknown & $16(24)$ \\
\hline \multicolumn{2}{|c|}{$\begin{array}{l}\text { *Except where noted otherwise. } \\
\text { †Demographic information for some nonphysician focus group participants was } \\
\text { not collected. }\end{array}$} \\
\hline
\end{tabular}

discussed in relation to theoretical saturation, where no new themes emerged. Data analyses were managed with the use of NVivo 10 software.

To minimize potential bias, the initial coding and analysis of the qualitative data were performed by 2 members of the qualitative team (R.F., S.D.S.) who were not involved in the quantitative study analysis. R.F., S.M.S. and S.D.S. were involved in the final stages of analysis as debriefers to provide credibility to the findings. All methodological decisions and insights were documented in an audit trail. ${ }^{14}$ Throughout analysis of transcripts, memos (detailed written accounts of events and decisions) were written to document the analysis process and facilitate identification of reasons for practice variation in the management of nephrotic syndrome. The transcripts and results were not circulated back to the participants for feedback following the viewpoint that this can be a threat to validity as the data have been analyzed and abstracted from the individual participants' perspective into themes from the data collected. ${ }^{17,18}$ Repeat interviews were not conducted.

\section{Ethics approval}

The study was approved by the Conjoint Health Research Ethics Board at the University of Calgary.

\section{Results}

Focus groups with 6-8 participants per group (total $n=67$ ) were conducted across 10 pediatric nephrology centres across Canada between March 2014 and April 2015. The focus groups lasted 40-60 minutes. A summary of centre and participant characteristics is given in Table 1 .

We grouped 5 emerging qualitative themes into centre and provider levels from the data (Figure 1). These themes were not derived in advance.

\section{Centre-level themes}

\section{Care model (shared v. individual)}

The type of care model (shared or individual) was discussed as an influencing factor for provider and team decision-making in

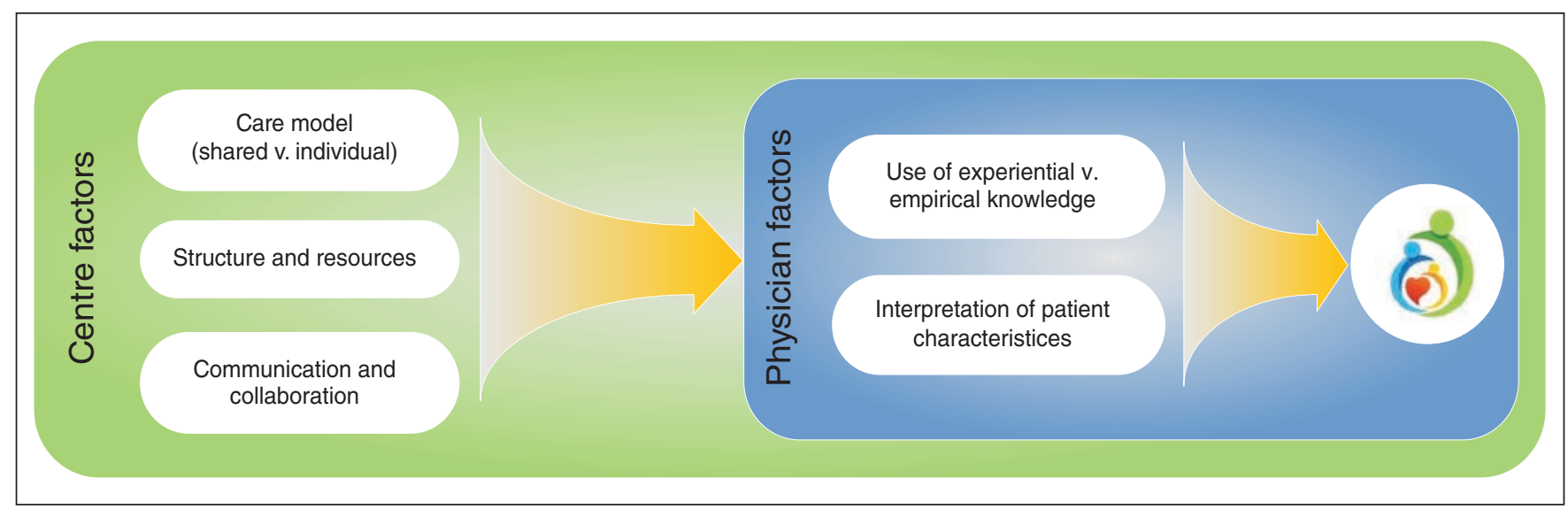

Figure 1: Themes generated during focus groups and how they interact with other to influence care variation in nephrotic syndrome. 
the management of nephrotic syndrome. Six of the 10 centres stated that they used a shared model of care for the management of nephrotic syndrome. With a shared model of care, patients did not have a single physician for their care management, and nursing teams coordinated care of patients depending on which physician was "on duty" for patients with nephrotic syndrome. Many of the physicians at centres with a shared model of care reported that such a care model reduces practice variation and creates a team approach to communication and decisionmaking for more complex management decisions. Some nurses stated that there are also negative consequences of this model of care on their workload and for patients' care experiences.

\section{Structures and resources}

The perceived degree of multidisciplinary involvement, financial implications of certain drug choices and system structures also influenced management decisions in nephrotic syndrome. All participating centres used a multidisciplinary approach, acknowledging dieticians, pharmacists and social workers as key players in education and management. Some centres, however, also discussed a lack of resources, which influenced practice variation in patient education, drug choices, monitoring and follow-up. The cost of second-line agents was also a common concern for participants across several centres. Medication coverage varies across the provinces of Canada, which influenced providers' and families' drug choices.

\section{Communication and collaboration}

A lack of communication and collaboration with other centres and/or community pediatricians (working in "silos") emerged as a major theme voiced by participants at all centres. Participants expressed that they were unaware of what protocols were being used at other centres, but some participants expressed that communication within their centre facilitated standardized practice. Nurses were viewed as having an important role in effective communication for nephrotic syndrome with parents, between physicians and between parents and physicians by providing consistent care and playing a major role in decision-making. Informants' quotations that represent the centre-level themes are shown in Table 2.

\section{Provider-level themes}

\section{Use of experiential versus empirical knowledge}

The providers who participated in our study used both experiential (tacit) and empirical knowledge to inform their decisionmaking in the treatment of nephrotic syndrome. Some providers reported relying more heavily on their experiential knowledge, whereas others relied more heavily on empirical knowledge to make treatment decisions.

For providers who were heavily guided by experiential knowledge, their place of training (key mentors), recent patient experiences, personal preferences and lack of trust in the current research evidence shaped decision-making in management. Other providers also voiced scepticism regarding the published evidence and doubted that anyone really knows how best to treat nephrotic syndrome. Challenges surrounding decision- making using empirical evidence in the context of scarce evidence emerged as discussion points. Decisions regarding tapering of steroids for initial presentation and relapses, choice of second-line agents and biopsy timing were heavily influenced by providers' experiential knowledge, which led to considerable variation in practice between providers. Many providers cited lack of research evidence to guide decisions in these areas.

Some providers mentioned that the use of research evidence influenced not only their individual practice but also the practice at their centre, stating that the use of research evidence standardized practice and reduced variation in individual provider practice. The KDIGO Clinical Practice Guideline for Glomerulonephritis ${ }^{3}$ was the most frequently mentioned empirical research guideline that influenced providers' decisions on the treatment of nephrotic syndrome. Not every participant stated that they used this guideline in practice.

\section{Interpretation of patient characteristics}

Providers' interpretation of patient and family characteristics and preferences emerged as factors influencing individualization of decision-making. Providers' decision-making was particularly influenced by the following patient characteristics: response to drug treatments, adherence to drug treatments, potential drug toxic effects and what that means to individual patients, ethnicity and family preferences. These factors influenced individual providers' decisions around length of treatment, medication tapering, choice of second-line drugs and timing of biopsy. However, many providers indicated that they present all treatment options to families and make a joint decision, given the potential efficacy and side effect profile. Drug toxic effects frequently emerged as an influencing factor in providers' treatment choices for their patients. Providers at some centres were likely to choose therapies with fewer toxic effects over therapies with more evidence and more toxic effects (e.g., avoiding cyclophosphamide because of the risk of infertility and cancer even though the drug has been shown to be effective in steroid-sensitive nephrotic syndrome). Informants' quotations that represent the provider-level themes are shown in Table 3.

\section{Interpretation}

In this qualitative study of pediatric nephrology care providers, we found that centre- and provider-level themes influenced practice variation. Providers reported using a combination of experiential and empirical knowledge to varying degrees in decision-making regarding treatment of children with nephrotic syndrome. The provider's judgement regarding the best course of action for the patient also played an important role in decision-making, especially in the setting of scarcity of evidence to guide many aspects of nephrotic syndrome treatment. Availability of resources, third-party coverage for steroid-sparing drugs, model of care in the nephrology clinic, and communication and collaboration within the multidisciplinary team were also important. These identified themes led to variations among providers within centres and between centres in steroid treatments, choice of second-line agents, and timing and frequency of biopsy in nephrotic syndrome care. 
Table 2: Representative key informant quotations on centre-level themes

\begin{tabular}{|c|c|}
\hline Theme & Quotation \\
\hline \multirow[t]{4}{*}{ Care model } & $\begin{array}{l}\text { We are becoming more consistent because we have gone to a shared } \\
\text { clinic model. I suspect we have become more consistent. I don't have } \\
\text { any data to prove that. We talk about it more, that we are going to } \\
\text { keep them on " } x \text { " dose. And the nurses are consistent, with the } 2 \\
\text { nurses. (Nephrologist } 1 \text {, site G) }\end{array}$ \\
\hline & $\begin{array}{l}\text { The parents find that very hard, that they see a different person every } \\
\text { time, sometimes. They find that a major challenge. There used to be a } \\
\text { doctor-based clinic. (Nurse } 2 \text {, site G) }\end{array}$ \\
\hline & $\begin{array}{l}\text { Shared clinics. So, yes, there is good advantage and yes, we do have } \\
\text { a good group and [for the] most part they all do the same thing. The } \\
\text { parents really, if it's somebody that is chronic and has an acute } \\
\text { episode, they would prefer seeing their regular physician than seeing } \\
\text { somebody else. (Nurse } 2 \text {, site } \mathrm{H} \text { ) }\end{array}$ \\
\hline & $\begin{array}{l}\text { I think the other thing to highlight is that we do have a shared clinic, so } \\
\text { that these nephrotics, right when they are coming to see us for } \\
\text { follow-up, it may be Dr. [name], it may be Dr. [name], it may be me. So } \\
\text { at least on Fridays, it is discussion. We have to come to a consensus } \\
\text { in a way, because whoever is going to see that person in clinic has to } \\
\text { be saying the same thing. So it works out well, those clinic rounds, in } \\
\text { conjunction with how we run the clinic. (Nephrologist } 6 \text {, site H) }\end{array}$ \\
\hline \multirow[t]{3}{*}{$\begin{array}{l}\text { Structures and } \\
\text { resources }\end{array}$} & $\begin{array}{l}\text { Cost might be an issue because provincially it varies from place to } \\
\text { place. If somebody said to us "Everyone is going to [mycophenolate } \\
\text { mofetil]," we would be, like, "Now you are dreaming." Or "Everybody } \\
\text { needs rituximab." We just couldn't do all of those things. (Nurse 1, } \\
\text { site B) }\end{array}$ \\
\hline & $\begin{array}{l}\text { If we are talking about workload, what affects workload is the absence } \\
\text { of dieticians and social workers. (Nurse } 1 \text {, site B) }\end{array}$ \\
\hline & $\begin{array}{l}\text { One of the things that I think was a barrier, and it's recently been } \\
\text { solved, is the cost and availability of the medications used to treat } \\
\text { nephrotic syndrome. You know, some of them can be very expensive } \\
\text { for families. (Nephrologist 2, site D) }\end{array}$ \\
\hline \multirow[t]{5}{*}{$\begin{array}{l}\text { Communication } \\
\text { and collaboration }\end{array}$} & $\begin{array}{l}\text { I'd have to say centre-specific. I trained in [site G], so you know, I know } \\
\text { what their protocol was - well, } 13 \text { years ago, I don't know what } \\
\text { they're currently using now. But I don't think there's any - I have no } \\
\text { idea what [site J] - you probably know what [site H] uses, because } \\
\text { you worked there. But I have no idea what other centres use. } \\
\text { (Nephrologist 2, site I) }\end{array}$ \\
\hline & $\begin{array}{l}\text { I guess I would say, if we read [investigator's] paper, we know there is } \\
\text { variability. I don't know if I could tell you what centre does what. } \\
\text { (Nephrologist } 1 \text {, site G) }\end{array}$ \\
\hline & $\begin{array}{l}\text { We don't care so much what everybody else is doing to tell you the } \\
\text { truth, because no one is really that convinced that they're ... } \\
\text { (Nephrologist 1, site C) } \\
\text { Because there's no solid evidence in anything, so ... (Nephrologist } 4 \text {, } \\
\text { site C) }\end{array}$ \\
\hline & $\begin{array}{l}\text { Yeah, reading, stuff like that. Actually, to be honest, I couldn't tell you } \\
\text { exactly how, yeah, how other centres treat and how they decide to } \\
\text { treat. We never had that communication, so it's like - it's funny } \\
\text { because nephrotic syndrome is probably one of the more common } \\
\text { diseases we see in pediatrics and pediatric nephrology. And I think the } \\
\text { assumption is that where you train is essentially how you're going to } \\
\text { practise. (Nephrologist } 1 \text {, site } \mathrm{J} \text { ) }\end{array}$ \\
\hline & $\begin{array}{l}\text { I think that our communication within the physicians is enhanced } \\
\text { greatly by having a dedicated nurse.... I think that's a great } \\
\text { enhancement to the communication between doctors and I think that } \\
\text { not having a dedicated nurse for nephrotic syndrome would be the } \\
\text { worst thing that could happen to us, if we did not have that. } \\
\text { (Nephrologist } 3 \text {, site E) }\end{array}$ \\
\hline
\end{tabular}


Table 3: Representative key informant quotations on provider-level themes

\begin{tabular}{|c|c|}
\hline Theme & Quotation \\
\hline \multirow[t]{3}{*}{$\begin{array}{l}\text { Use of experiential } \\
\text { knowledge }\end{array}$} & $\begin{array}{l}\text { Depends on where you trained and how much of the literature you believe } \\
\text { or you think is more relevant. (Nephrologist } 4 \text {, site C) }\end{array}$ \\
\hline & $\begin{array}{l}\text { The problem is that there is not a lot of good evidence in nephrotic } \\
\text { syndrome. (Nephrologist } 3 \text {, site } \mathrm{H} \text { ) }\end{array}$ \\
\hline & $\begin{array}{l}\text { I think we are regrettably influenced by whatever happened to our } \\
\text { patients. So if you miss something, nobody wants to feel, like, "Oh, I } \\
\text { missed that diagnosis." Even though it might be not, maybe it is super } \\
\text { rare, that's never going to happen again to anyone in North America, the } \\
\text { fact that it happened you, it somehow influences, it strongly influences the } \\
\text { way you manage the next patient. I think your own personal experience is } \\
\text { the trump card of all, regrettably whether that's evident and it is not } \\
\text { evidence based. (Nephrologist } 4 \text {, site E) }\end{array}$ \\
\hline \multirow[t]{2}{*}{$\begin{array}{l}\text { Use of empirical } \\
\text { knowledge }\end{array}$} & $\begin{array}{l}\text { We developed the guidelines, because everybody has their own recipe, } \\
\text { and I think we all acknowledge that from the outset, and we really wanted } \\
\text { to, as much as possible, base it on available evidence rather than base it } \\
\text { on, you know, somebody's favourite recipe. (Nephrologist } 1 \text {, site B) }\end{array}$ \\
\hline & $\begin{array}{l}\text { I mean, there were the KDIGO guidelines that came out for the treatment } \\
\text { of nephrotic syndrome a couple years ago, and I think a review of our } \\
\text { treatment protocols would suggest that we're actually right online with } \\
\text { what they recommend. (Nephrologist } 2 \text {, site A) }\end{array}$ \\
\hline \multirow[t]{3}{*}{$\begin{array}{l}\text { Interpretation of } \\
\text { patient } \\
\text { characteristics }\end{array}$} & $\begin{array}{l}\text { The reality of the nephrotics is that there are going to be some subgroups } \\
\text { of people where there is need to modify your simple original prescription } \\
\text { because they don't respond in the way you expected them to. } \\
\text { (Nephrologist } 1 \text {, site E) }\end{array}$ \\
\hline & $\begin{array}{l}\text { I think there is a variability component that has to do with patient } \\
\text { adherence as well, in the sense that if they are either experiencing toxicity } \\
\text { or they don't believe in the therapy, or they have different ideas, whatever } \\
\text { it is, they may wean themselves in a different way from what was } \\
\text { prescribed, which is still a variation in therapy. (Nephrologist } 1 \text {, site B) }\end{array}$ \\
\hline & $\begin{array}{l}\text { I tend to use [mycophenolate mofetil] as a first-line for my steroid-sparing } \\
\text { because it doesn't have nephrotoxicity and sometimes it works. } \\
\text { (Nephrologist 4, site E) }\end{array}$ \\
\hline
\end{tabular}

One of the most significant factors influencing provider decision-making in nephrotic syndrome is the varying use of experiential and empirical knowledge by providers. Use of experiential evidence, or tacit knowledge, was important to many owing to the challenge of providing care when there is a lack of strong research evidence to guide management. ${ }^{19}$ The evidence supporting many guideline recommendations for steroid therapy and second-line agents in pediatric nephrotic syndrome is based on small numbers of prospective randomized trials, some with very few patients, which leads to lack of trust among the provider groups in the robustness of the literature. ${ }^{3,5}$ This uncertainty regarding evidence may be leading to physicians' heavy reliance on tacit knowledge gained from training and past experience to provide care. Nonetheless, existing and emerging evidence was also used to varying degrees in decisionmaking. This study, therefore, provides some understanding of the cognitive strategies used by pediatric nephrology providers in day-to-day clinical practice when treatment decisions are made in complex, ambiguous clinical situations with uncertain outcomes. ${ }^{20}$ These findings have implications for translation and adoption of new knowledge into pediatric nephrology practice and overall improvement in quality of care.
The second theme we elicited in the focus groups related to providers' judgement regarding patient/family needs and preferences when making treatment decisions. A key driver of selection of best treatment involved known and potential toxic effects of the drugs used. As the current findings confirm, there are strong opinions regarding what therapy is best, ${ }^{7}$ and some therapies such as cyclophosphamide (with higher grades of evidence of efficacy in steroid-sensitive nephrotic syndrome) were frequently passed over as a steroid-sparing agent in some centres in favour of other drugs with less empirical evidence but fewer toxic effects. Given these paradoxical findings, the weight given to patient preference and provider experiential knowledge during shared medical decision-making is vague. Patient-centred decision-making under complex and ambiguous conditions requires awareness of the unique characteristics of each clinical situation, including patient demographic characteristics, comorbidities, literacy, values and beliefs. Fully informed patients are more likely to participate in care, make wiser decisions, come to common understanding with providers and adhere to therapy, ${ }^{21,22}$ and the effect of provider preferences regarding drug selection on patient outcomes is unknown. 
Shared-care models (where 1 patient was looked after by many providers in a single clinic) evoked mixed responses from participants, and further work is necessary to determine whether such models provide best patient-centred care. Provider groups communicated within their centre, but there were no efforts to discuss care protocols nationally. Protocols were adapted from 1 centre to another as trainees moved on and were periodically changed or overhauled based on emerging evidence and by the efforts of a champion at each centre. There was a certain apathetic feeling: "No one really knows best treatment anyway, so why consult other centres?" This disconnectedness among pediatric nephrology providers has perhaps the easiest solution: creating forums for sharing protocols at regular intervals where provider groups have time to reflect on their own practice and modify practice based on best available evidence and consensus among national and international colleagues.

\section{Strengths and limitations}

We gathered a large set of qualitative data by conducting focus groups in $77 \%$ of all pediatric nephrology centres in Canada. The focus group data may be limited by the composition of the participants; however, the moderator used interviewing techniques that enabled participation by all members of the focus groups.

\section{Conclusion}

We found that both centre- and provider-level factors influenced the care of children with nephrotic syndrome. Many providers relied on experiential evidence and consultation with colleagues within their own centres to inform practice. Providers' perceptions of drug toxic effects and what may be best for each patient based on patient and family characteristics, strongly shaped by experiential knowledge, influenced many of the treatment choices for second-line agents and biopsy practices.

\section{References}

1. Eddy AA, Symons JM. Nephrotic syndrome in childhood. Lancet 2003;362: 629-39.

2. Gipson DS, Massengill SF, Yao L, et al. Management of childhood onset nephrotic syndrome. Pediatrics 2009;124:747-57.

3. KDIGO Clinical Practice Guideline for Glomerulonephritis. Kidney Int Suppl 2012;2:143-53.

4. Hodson EM, Willis NS, Craig JC. Corticosteroid therapy for nephrotic syndrome in children. Cochrane Database Syst Rev 2007;(4):CD001533.

5. Pasini A, Aceto G, Ammenti A, et al. Best practice guidelines for idiopathic nephrotic syndrome: recommendations versus reality. Pediatr Nephrol 2015;30: 91-101.

6. MacHardy N, Miles PV, Massengill SF, et al. Management patterns of childhood-onset nephrotic syndrome. Pediatr Nephrol 2009;24:2193-201.

7. Samuel S, Morgan CJ, Bitzan M, et al. Substantial practice variation exists in the management of childhood nephrotic syndrome. Pediatr Nephrol 2013;28: 2289-98.

8. Fujinaga S, Endo A, Ohtomo Y, et al. Uncertainty in management of childhoodonset idiopathic nephrotic syndrome: is the long-term prognosis really favorable? Pediatr Nephrol 2013;28:2235-8.

9. Zurynski Y, Frith K, Leonard H, et al. Rare childhood diseases: How should we respond? Arch Dis Child 2008;93:1071-4

10. Logan J, Graham ID. Toward a comprehensive interdisciplinary model of health care research use. Sci Commun 1998;20:227-46.

11. Graham ID, Logan J, Harrison MB, et al. Lost in knowledge translation: Time for a map? 7 Contin Educ Health Prof 2006;26:13-24.

12. Lehoux P, Poland B, Daudelin G. Focus group research and "the patient's view." Soc Sci Med 2006;63:2091-104.
13. Kitzinger J. The methodology of focus groups: the importance of interaction between research participants. Sociol Health Illn 1994;16:103-21.

14. Morse JM, Field PA. Qualitative research methods for health professionals. 2nd ed. Thousand Oaks (CA): Sage; 1995.

15. Scott SD, Sharpe H, O'Leary K, et al. Court reporters: A viable solution for the challenges of focus group data collection? Qual Health Res 2009;19:140-6.

16. Patton MQ. Qualitative research and evaluation methods. 2 nd ed. Thousand Oaks (CA): Sage; 2001.

17. Morse JM. Validity by committee [editorial]. Qual Health Res 1998;8:443-5.

18. Morse JM, Barrett M, Mayan M, et al. Verification strategies for establishing reliability and validity in qualitative research. Int Qual Methods 2002;1:13-22.

19. Croskerry P. From mindless to mindful practice - cognitive bias and clinical decision making. N Engl 7 Med 2013;368:2445-8.

20. Epstein RM. Mindful practice. 7AMA 1999;282:833-9.

21. Barry MJ, Edgman-Levitan S. Shared decision making - pinnacle of patientcentered care. N Engl 7 Med 2012;366:780-1.

22. Charles C, Gafni A, Whelan T. Shared decision-making in the medical encounter: What does it mean? (Or it takes at least two to tango). Soc Sci Med 1997;44:681-92.

Affiliations: Section of Nephrology (Samuel, Wade), Department of Pediatrics, Cumming School of Medicine, University of Calgary, Calgary, Alta.; Faculty of Nursing (Flynn), University of Alberta, Edmonton, Alta.; Division of Nephrology (Zappitelli), Department of Pediatrics, McGill University Health Centre, Montréal, Que.; Division of Nephrology (Dart, Pinsk), Department of Pediatrics, College of Medicine, University of Manitoba, Winnipeg, Man.; Division of Nephrology (Parekh), Department of Pediatrics, University of Toronto, Toronto, Ont.; Division of Nephrology (Mammen), Department of Pediatrics, Faculty of Medicine, University of British Columbia, Vancouver, BC; Faculty of Nursing (Scott), Edmonton Clinic Health Academy, University of Alberta, Edmonton, Alta.

Contributors: Susan Samuel, Shannon Scott and Rachel Flynn participated in data acquisition, analysis and interpretation. Susan Samuel and Rachel Flynn drafted the manuscript. All of the authors participated in the conception and design of the study, critically reviewed the manuscript for important intellectual content, approved the final version to be published and agreed to act as guarantors of the work. The other members of the Canadian Childhood Nephrotic Syndrome Project Team contributed to facilitation of focus groups at individual sites.

Funding: This project was funded by operating grants from the Roy and Vi Baay Chair in Kidney Research, University of Calgary, the Kidney Foundation of Canada (grant KFPC140020) and the Canadian Institutes of Health Research (grant PNI-134070). Susan Samuel was supported by salary awards from the Kidney Foundation of Canada KRESCENT Program and the Canadian Child Health Clinician Scientist Program. Rachel Flynn is supported by the Alberta Innovates - Health Solutions Clinician Fellowship and the Women \& Children's Health Research Institute, University of Alberta. Michael Zappitelli is supported by a research salary award from the Fonds de recherche du Québec - Santé. Shannon Scott is supported by a Canada Research Chair (Tier 2) and an Alberta Innovates Health Solutions Population Health Investigator Award.

Acknowledgement: The authors acknowledge all the pediatric nephrology care providers who gave of their time to participate in the focus groups.

Additional members of the Canadian Childhood Nephrotic Syndrome Project Team: Allison Eddy, University of British Columbia, Vancouver, BC; Catherine Morgan, University of Alberta, Edmonton, Alta.; Alberto Nettel-Aguirre, University of Calgary, Calgary, Alta.; Silviu Grisaru, University of Calgary, Calgary, Alta.; Robin Erickson, University of Saskatchewan, Saskatoon, Sask.; Guido Filler, Western University, London, Ont. Steven Arora, McMaster University, Hamilton, Ont.; Janusz Feber, University of Ottawa, Ottawa, Ont; Pavel Geier, University of Ottawa, Ottawa, Ont.; Martin Bitzan, McGill University, Montréal, Que.; Geneviève Benoit, Centre hospitalier universitaire Sainte-Justine, Université de Montréal, Montréal, Que.; Anne-Laure Lapeyraque, Centre hospitalier universitaire Sainte-Justine, Université de Montréal, Montréal, Que.; Colette Girardin, Centre hospitalier universitaire de Sherbrooke, Sherbrooke, Que.; James Tee, Dalhousie University, Halifax, NS

Supplemental information: For reviewer comments and the original submission of this manuscript, please see www.cmajopen.ca/content $/ 5 / 2 /$ E424/suppl/DC1 